\title{
WNN in Image Restoration for Industrial Applications
}

\author{
Anoopa Jose Chittilappilly \\ Electronics \& Communication Engineering \\ Department, \\ Karpagam Academy of Higher Education, \\ Coimbatore,Tamil Nadu, India.
}

\author{
Kamalraj Subramaniam \\ Electronics \& Communication Engineering \\ Department, \\ Karpagam Academy of Higher Education, \\ Coimbatore,Tamil Nadu, India.
}

\begin{abstract}
In many applications, such as astronomy, remote sensing, medical imaging, military detection, public security, and video technology, images are the main sources of information. But, due to some reasons, observed images are degraded. The degradations are mainly caused by blur and noise. The aim of image restoration is to obtain restored image which should be as close as the original image. Wavelet transforms and neural networks have proven to be very efficient and effective in analyzing a very wide class of signals and phenomena. Wavelet expansion allows a more accurate local description and separation of signal characteristics. Here image processing is introduced for industrial applications in automatic visual inspection system because visual inspection system is not able to identify the small flaws in the industrial products.
\end{abstract}

\section{Keywords}

Image Restoration, Degradation model, Wavelet Neural Network

\section{INTRODUCTION}

In many applications, the measured images are degraded by blurs; e.g. the optical system in a camera lens may be out of focus, so that the incoming light is smeared out, and in astronomical imaging the incoming light in the telescope has been slightly bent by turbulence in the atmosphere. In addition, images that occur in practical applications inevitably suffer from noise, which arise from numerous sources such as radiation scatter from the surface before the image is sensed, electrical noise in the sensor or camera, transmission errors and bit errors as the image is digitized etc. In image formation, the observed images are usually blurred by optical instruments and/or transfer medium and contaminated by noise, which makes image restoration a classical problem in image processing.

In this paper, an automatic system is proposed for detecting the presence of split defects in sheet-metal forming processes. An automated visual inspection system contributes significantly to guarantee 100 percent of quality parts, reducing costs, and increased the competitiveness, but the visual inspection of complex shaped metallic surfaces is extremely difficult. Sheet-metal parts produced in forming processes are a challenge to any vision system where their non-lambertian surfaces can produce saturations, shadows, inter-reflections, and other quick variations that are extremely difficult to predict. Another difficulty is that they tend to have edges, corners, moldings, creases, etc., which may produce signals which are similar to that of a defect.

In an existing system they used an appearance-based approaches proposed by Murase and Nayer [1].The problem of using appearance based approach is that there will be a significant off-line costs. Another problem considering in the existing method is it depends upon the bright. Due to the charge coupled device and diffuse illumination system sometimes perfect size is not achievable. In obtainable method the whole device is handled by robots since there will be a time management. So we have to enhance the system in an efficient way.

\section{DEGRADATION MODELS}

A general model for image degradation that represents these processes is:

$$
\mathrm{d}(\mathrm{x}, \mathrm{y})=\sum_{\mathrm{i}, \mathrm{j}}^{\mathrm{x}} \mathrm{h}(\mathrm{x}-\mathrm{i}, \mathrm{y}-\mathrm{j}) \mathrm{f}(\mathrm{x}, \mathrm{y})+\mathrm{\eta}(\mathrm{x}, \mathrm{y})(1)
$$

where $h(x-i, y-j)$ is the two dimensional impulse response of the blurring system at pixel $(\mathrm{x}, \mathrm{y})$, represent a point-wise operation, and $\eta(\mathrm{x}, \mathrm{y})$ is the corruptive noise process. It is usually assumed that the blurring function is space-invariant and the noise $\eta(\mathrm{x}, \mathrm{y})$ is the additive white Gaussian process. As the blur function is assumed space-invariant so the nonlinear degradation process becomes linear degradation process. However, in some applications, it is necessary to take the sensor non linearity into account.

\section{RELATED WORKS}

\subsection{Wavelet Based Sparse Reduced-Rank Regression for Hyper Spectral Image Restoration}

Rasti B. et al [2] presented a method called waveletbased sparse reduced-rank regression (WSRRR) was implemented to hyper spectral image restoration. The method was based on minimizing a sparse regularization problem subject to an orthogonality constraint. A cyclic descent-type algorithm was derived for solving the minimization problem. For selecting the tuning parameters, implemented the method based on Stein's unbiased risk estimation. It was shown that the hyper spectral image can be restored using a few sparse components. This method was evaluated using signal-to-noise ratio and spectral angle distance for a simulated noisy data set and by classification accuracies for a real data set. Two different classifiers, namely, support vector machines and random forest, were used in this approach. This method was compared to other restoration methods, and it was shown that WSRRR outperforms the simulated noisy data set. It was also shown in that the experiments on a real data set that WSRRR not only effectively removes noise but also maintains more fine features compared to other methods used. WSRRR also gives higher classification accuracies.

\subsection{Generalized Multivariate Exponential Power Prior for Wavelet-Based Multichannel Image Restoration}

In multichannel imaging, several observations of the same scene acquired in different spectral ranges are available. Very 
often, the spectral components are degraded by a blur modeled by a linear operator and an additive noise. In this Marnissi et al [3], addressed the problem of recovering the image components in a wavelet domain by adopting a variational approach. Approach to the addressed method was in twofold. First, an appropriate multivariate penalty function was derived from a novel joint prior model of the probability distribution of the wavelet coefficients located at the same spatial position in a given sub band through all the channels. Secondly, the challenging issue of computing the Maximum a Posteriori estimate by using a Majorize-Minimize optimization strategy is addressed. Simulation tests were carried out on multispectral satellite images showed that the proposed method outperforms conventional techniques.

\subsection{A New Algorithm for Infrared Image Restoration Based on Multi-Scale Morphological Wavelet and Hopfield Neural Network}

Based on the complexity and randomness of the infrared image degradation factors, and integrates the strong de-noising features of multi-scale morphological wavelet and the salient problem solving features of Hopfield neural network in optimization. In this Ao-Chang Pan et al [4] presented a new algorithm for infrared degraded image restoration. Algorithm takes advantage of continuous recycle between multi-scale morphological wavelet denoising and Hopfield neural network iteration so as to makes access to a better recovery of infrared images. This algorithm also solved the problems in noise suppression and image detail protection of traditional Hopfield neural network image restoration algorithm and successfully protects the edge of the recovery images and details. Simulation results proved the effectiveness of the recovery algorithm.

\subsection{A Fast Wavelet-Based Reconstruction Method for Magnetic Resonance Imaging}

In this approach, the fact that wavelets can represent magnetic resonance images well, with relatively few coefficients is taken into account. This property is used to improve MRI reconstructions from under sampled data with arbitrary kspace trajectories. Reconstruction was posed as an optimization problem that could be solved with the iterative shrinkage/thresholding algorithm (ISTA) which, unfortunately, converges slowly. To make the approach more practical, Guerquin-Kern et al [5] proposed a variant that combines recent improvements in convex optimization and that can be tuned to a given specific k-space trajectory presented a mathematical analysis that explains the performance of the algorithms. Using simulated and in vivo data showed that the nonlinear method is fast, as it accelerates ISTA by almost two orders of magnitude. It is shown that it remains competitive with TV regularization in terms of image quality.

\section{FUNDAMENTALS OF WAVELET TRANSFORM}

Wavelet transforms (WT) provide the alternative to the shorttime Fourier transform (STFT) for non-stationary signal analysis. Both STFT and WT result in signal decomposition into two-dimensional function of time and frequency respectively scale. The basic difference between these two transforms is in the construction of the window function which has a constant length in the case of the STFT (including rectangular, Blackman and other window functions) while in the case of the WT wide windows are applied for low frequencies and short windows for high frequencies to ensure constant time-frequency resolution. Local and global signal analysis can be combined in this way.

Both wavelet theory and wavelet-based image restoration are complex and evolving subjects. Wavelets are a mathematical tool for representing and approximating functions hierarchically. The wavelet transform is similar to a Fourier transform in the sense that it decomposes a signal into a set of basis functions. In the case of the Fourier transform this basis consists of sine and cosine functions at different frequencies. In the wavelet transform, there is a single function $\psi$, called the mother wavelet. Any function can be represented by superimposing dilated and translated versions of $\psi$, denoted by $\psi_{\mathrm{a}, \mathrm{b}}$, where $\mathrm{a}$ and $\mathrm{b}$ are the dilation and translation parameters[6]. The $\psi_{\mathrm{a}, \mathrm{b}}$ can be computed from the mother wavelet as

$\psi_{\mathrm{a}, \mathrm{b}}(\mathrm{x})=\frac{1}{\sqrt{\mathrm{a}}} \psi\left(\frac{\mathrm{x}-\mathrm{b}}{\mathrm{a}}\right), \mathrm{a} \in \mathrm{R}^{+}, \mathrm{b} \in \mathrm{R}$

where each wavelet function in the decomposition is formed from a mother wavelet function $\psi(\mathrm{x})$ which is scaled (given by a) and translated (b),the result being localized in both the frequency and spatial domains. The process of decomposing a function into wavelet coefficients (a scaling factor for each of the $\psi_{\mathrm{a}, \mathrm{b}}$ ) is called wavelet transform[6]. If the parameters a and $\mathrm{b}$ take on discrete values, we have a discrete wavelet transform or DWT, essentially leading to a finite number of coefficients.

For two dimensional signals, the conventional DWT may be regarded as equivalent to filtering the input signal with a bank of band pass filters, whose impulse responses are all approximately given by scaled versions of a mother wavelet. The scaling factor between adjacent filters is usually two to one (2:1), leading to octave bandwidths and center frequencies that are one octave apart. At the coarsest scale, a low pass filter is also required to represent the lowest frequencies of the signal. In other words wavelet analysis is a version of windowing technique, but with varying window size. It allows the use of longer windows when more precise low frequency information is required and shorter windows where high frequency information is needed. WT in wavelet analysis is analogous to the Fourier Transform in Fourier analysis.

Wavelet dilation corresponds to spectrum compression. The most common choice includes Daubeshie's wavelets even though their frequency characteristics stand for approximation of band-pass filters only. On the other hand harmonic wavelets can have broader application in many engineering problems owing to their very attractive spectral properties.

Information about signals resulting from a selected process can be based upon signal decomposition by a given set of wavelet functions into separate levels or scales resulting in the set of wavelet transform coefficients. These values can be used for signal compression, signal analysis, segmentation and in the case that these coefficients are not modified they allow the following perfect signal reconstruction. In the case that only selected levels of signal decomposition are used or wavelet transform coefficients are processed it is possible to extract signal components or to reject its undesirable parts.

Using the threshold method it is further possible to reject noise and to enlarge signal to noise ratio. The de-noising 
algorithm assumes that the signal contains low frequency components and it is corrupted by the additive Gaussian white noise with its power much lower than power of the analyzed signal. The whole method consists of the following steps:

- Signal decomposition using a chosen wavelet function up to the selected level and evaluation of wavelet transform coefficients

- The choice of threshold limits for each decomposition level and modification of its coefficients

- $\quad$ Signal reconstruction from modified wavelet transforms coefficients.

Results of this process depend upon the proper choice of wavelet functions, selection of threshold limits and their use..

\section{NEURAL NETWORK}

One efficient way of solving complex problems is following the lemma "divide and conquer". A complex system may be decomposed into simpler elements, in order to be able to understand it. Also simple elements may be gathered to produce a complex system. Networks are one approach for achieving this. There are a large number of different types of networks, but they all are characterized by the following components: a set of nodes, and connections between nodes. The nodes can be seen as computational units. They receive inputs, and process them to obtain an output. This processing might be very simple (such as summing the inputs), or quite complex (a node might contain another network).The connections determine the information flow between nodes. They can be unidirectional, when the information flows only in one sense, and bidirectional, when the information flows in either sense. The interactions of nodes though the connections lead to a global behaviour of the network, which cannot be observed in the elements of the network. This global behaviour is said to be emergent. This means that the abilities of the network supersede the ones of its elements, making networks a very powerful tool. One type of network sees the nodes as 'artificial neurons'. These are called artificial neural networks (ANNs).

An artificial neuron is a computational model inspired in the natural neurons [7]. These basically consist of inputs (like synapses), which are multiplied by weights (strength of the respective signals), and then computed by a mathematical function which determines the activation of the neuron. Another function (which may be the identity) computes the output of the artificial neuron (sometimes in dependence of a certain threshold). ANNs combine artificial neurons in order to process information. The higher a weight of an artificial neuron is, the stronger the input which is multiplied by it will be. Weights can also be negative, so we can say that the signal is inhibited by the negative weight. Depending on the weights, the computation of the neuron will be different [8]. By adjusting the weights of an artificial neuron we can obtain the output we want for specific inputs. But when we have an ANN of hundreds or thousands of neurons, it would be quite complicated to find by hand all the necessary weights. But we can find algorithms which can adjust the weights of the ANN in order to obtain the desired output from the network.

\section{IMAGE RESTORATION USING WNN}

\section{In this Wavelet neural network (WNN) is presented for Image} restoration. It is the operation of taking a corrupted image and estimating the clean original image. With the help of wavelet neural network, a new image restoration algorithm is proposed for restoring and for resizing the image amoeba element used. Image data is extracted by amoeba structure element, and input into the wavelet neural network. The WNN is trained with the obtained model in batch mode training, which adjusts the wavelet coefficient and network weights adaptively to get the restored image. Then it is compared with the original image for defect identification. The experimental results will show that the approach proposed can preserve fine details and excellent fidelity and is better than general denoising methods. WNN is introduced into the field of image restoration due to the excellent local feature and adaptive ability. The WNN has better approximation performance and also have the better restoration performance.

In this architecture model degraded image is given as the input to the amoeba operator. It is a new type of dynamic structure elements is designed according to the physical structure of amoeba-amoeba structure element. It is a kind of filter which can automatically adjust shapes and sizes of structure elements according to the content of the image. The resized image is classified into flat and edge parts as the training vector pairs. These training vector pairs are given to the Wavelet Neural Network. Divide the degraded images into flat and edge parts by amoeba operator. Then train the models of vector pairs of both neural network model and degraded image model. From these training models original image estimation is obtained. Then it is compared with the original image for problem recognition. A brand new sort of vibrant design aspects was made good actual design involving amoeba- amoeba design component. This is a sort of separate out which often can on auto-pilot change sizes and heights according to the content of the image. The shape of amoeba structure element is obtained by amoeba distance calculation, which is different from stationary structure element, and the length of path $\sigma$ is defined as follows

$$
L(\sigma)=\sum_{i=0}^{n-1}\left[1+\lambda_{1} d_{\text {pixel }}\left(x_{i}, x_{i+1}\right)\right]
$$

In which, $\mathrm{d}_{\text {pixel }}\left(\mathrm{x}_{\mathrm{i}}, \mathrm{x}_{\mathrm{i}+1}\right)$ is the distance between image pixel point $\mathrm{x}_{\mathrm{i}}$ and image pixel point $\mathrm{x}_{\mathrm{i}+1}$ and $\sigma=\left(\mathrm{x}=\mathrm{x}_{0}, \mathrm{x}_{1}, \ldots, \mathrm{x}_{\mathrm{n}}\right.$ ${ }_{1}, \mathrm{x}_{\mathrm{n}}=\mathrm{y}$ )is the path between the point $\mathrm{x}_{\mathrm{i}}$ and $\mathrm{x}_{\mathrm{i}+1}$.

Between pixel point $\mathrm{x}$ and $\mathrm{y}, \mathrm{n}$ is the number of path $(\sigma)$. Because $\lambda_{1}$ is a real number, the amoebas distance with parameter $\lambda_{1}$ is defined as follows,

$$
\left\{\begin{array}{l}
\mathrm{d}_{\lambda_{1}}(\mathrm{x}, \mathrm{x})=0 \\
\mathrm{~d}_{\lambda_{1}}(\mathrm{x}, \mathrm{y})=\min \mathrm{L}(\sigma)
\end{array}\right.
$$

There's a robust relationship between geometric ranges expressed by means of amoebas range along with the dull range of image pixel stage. Next while using the regulation pertaining to parameter $\lambda_{1}$ image gradient info can be expressed clearly. This amoeba framework factor has become understood to be follow:

$$
\operatorname{Amoeba}(x)=\left\{y \mid d_{\lambda_{1}}(x, y) \leq r\right\}
$$

In which, $r$ is a real number and is the radius of amoeba structure element, through adjusted the $r$, An amoeba-like structure elements can be divided into different scales, This is like from center pixels to start the near-far distinguish different levels, And each layer has different weights, the weights of the different scales are defined as follows: 


$$
w_{i}=\eta \frac{1 / b_{i}}{\sum_{j=1}^{r} 1 / b_{j}} i=1,2 \ldots . r
$$

Constructing the amoeba structure element with those equations will help to place any industrial parts or any type of images with suitable size and shapes corresponding to their specifications and the element will provide the better utilization for the purposes of image restoration.

$\mathrm{X}_{1}^{\mathrm{p}}, \mathrm{X}_{\mathrm{k}}^{\mathrm{p}}$ are the input vectors, $\mathrm{y}_{1}^{\mathrm{p}}, \mathrm{y}_{\mathrm{i}}^{\mathrm{p}}$ are the predicted output, $\mathrm{w}_{\mathrm{jk}}, \mathrm{w}_{\mathrm{ij}}$ are the weights connecting every layer. $\Psi_{1}, \Psi_{2}$ are the mother wavelet function. The steps involved in the training process of WNN are as follows:-

Step 1: Data preprocessing: first, the original data is quantified and normalized, and then the data is divided into training set and testing set for network training and testing, respectively.

Step 2: Initializing WNN: connection weights and, translation factor, and scale factor are randomly initialized, and the learning rate is set.

Step 3: Training network: input the training set into WNN, compute network predicted output values, and calculate the error between output and the expected value.

Step 4: Updating the weights: update mother wavelet function parameters and network weights according to the prediction error, making the predictive value of the network as close to actual values.

Step 5: If the results satisfy the given conditions, use the testing set to test the network, otherwise, return to Step 3.

After initializing the WNN using connection weights, translation factor, scale factor are randomly initialized and the learning rate is set. The used WNN in this paper has the following form,

$$
\Psi_{\text {aj } b j}\left(\operatorname{net}_{j}^{\mathrm{p}}(\mathrm{t})\right)=\Psi\left[\frac{\operatorname{net}_{\mathrm{j}}^{\mathrm{p}}(\mathrm{t})-\mathrm{b}_{\mathrm{j}}(\mathrm{t})}{\mathrm{a}_{\mathrm{j}}(\mathrm{t})}\right]
$$

$\Psi$ is the mother wavelet and assuming that there exists a denumerable family derived from $\Psi$. Let $a_{j}(t), b_{j}(t)$ are the scale and translation parameters.

$$
\text { where, } \operatorname{net}_{j}^{\mathrm{p}}(\mathrm{t})=\sum_{\mathrm{k}=1}^{\mathrm{m}} \mathrm{w}_{\mathrm{jk}}(\mathrm{t}) \mathrm{x}_{\mathrm{k}}(\mathrm{t}) \mathrm{a}
$$

$\mathrm{w}_{\mathrm{jk}}$ is the wavelet coefficients of the expansion that constitute the discrete set.Computing the network predicted output values and calculating the error between output and expected value using the following relation.

$$
\begin{aligned}
& Y_{i}^{p}(t)=f\left[\sum_{j=1}^{N} w_{j k}(t) \Psi_{a j b j}\left(\sum_{k=1}^{m} w_{j k}(t) x_{k}^{p}(t)\right)\right] \\
& E(t)=\frac{1}{2} \sum_{p=1}^{m} \sum_{i=1}^{n}\left[d_{i}^{p}-Y_{i}^{p}\right] 2
\end{aligned}
$$

Updating the weights,updating mother wavelet function parameter and network weights acording to the prdiction error that we have obtained. The wavelet function used in this paper as follows.,
$\Psi(\mathrm{x})=\left(1-\frac{\mathrm{x}^{2}}{\varepsilon^{2}}\right) \exp \left(-\frac{\mathrm{x}^{2}}{2 \varepsilon^{2}}\right)$

After setting the initial weights of the network and inputting training samples, the output of wavelet network can be calculated by using the current neural network parameters. It can optimize the network through the following formula for making the predicted value of the network as close to the actual value. Optimizing the network for input to hidden layer,

$$
\begin{aligned}
& \frac{\partial E(t)}{\partial w_{j k}(t)}= \\
& \frac{\sum_{p=1}^{m} \sum_{i=1}^{n}\left(d_{i}^{p}-y_{i}^{p}(t)\right) w_{i j}(t) \Psi_{a j, b j}^{\prime}\left(\operatorname{net}_{j}^{p}(t)\right) k_{k}^{p}(t)}{a_{j}(t)}
\end{aligned}
$$

Adjusting the network for hidden layer to output layer,

$$
\frac{\partial E(t)}{\partial w_{i j}(t)}=\sum_{p=1}^{m}\left(d_{i}^{p}-y_{i}^{p}(t)\right) \Psi \text { aj, bj }\left(\operatorname{net}_{j}^{p}(t)\right)
$$

For making the predicted value close to actual value scale and translation parameters also optimized using the following formula

$$
\begin{aligned}
& \frac{\partial E(t)}{\partial_{a j}(t)}= \\
& \frac{\sum_{p=1}^{m} \sum_{i=1}^{n}\left(d_{i}^{p}-y_{i}^{p}(t)\right) w_{i j}(t) \Psi_{a j, b j}^{\prime} \frac{\left[\left(\operatorname{net}_{j}^{p}(t)\right)-b_{j}(t)\right]_{j}^{p}(t)}{a_{j}(t)}}{a_{j}(t)} \\
& \frac{\frac{\partial E(t)}{\partial_{b j}(t)}=}{\sum_{p=1}^{m} \sum_{i=1}^{n}\left(d_{i}^{p}-y_{i}^{p}(t)\right) w_{i j}(t) \Psi_{a j, b j}^{\prime}\left(\operatorname{net}_{j}^{p}(t)\right)}
\end{aligned}
$$

The parameters of wavelet neural network is adjusted by the following formula [9],

$w_{j k}(t+1)=w_{j k}(t)-\eta \frac{\partial E(t)}{\partial w_{j k}(t)}+\mu \Delta w_{j k}(t)$

$\mathrm{w}_{\mathrm{jk}}$ is the wavelet coefficients of the expansion that constitute the discrete set. $\Delta$ is the gradient of the objective function. Assuming that $\mathrm{w}$ is the connection weight of the output layer adjusting parameter in a node, the generally used learning rule is [9]

$w_{i j}(t+1)=w_{i j}(t)-\eta \frac{\partial E(t)}{\partial w_{i j}(t)}+\mu \Delta w_{i j}(t)$

$a_{j}(t), b_{j}(t)$, are the scale and translation parameters, $\eta$ is the learning rate and updating the parameters $a_{j}(t), b_{j}(t)$.

$a_{j}(t+1)=a_{j}(t)-\eta \frac{\partial E(t)}{\partial a_{j}(t)}+\mu \Delta a_{j}(t)$
$b_{j}(t+1)=b_{j}(t)-\eta \frac{\partial E(t)}{\partial b_{j}(t)}+\mu \Delta b_{j}(t)$

Where $\eta$ represents the learning rate of wavelet neural network, $\mu$ represents the momentum coefficient. Thus 
obtained WNN based on mathematical morphology will be used to training the samples that obtained from the degraded image to restore the original image from the degraded image.

\section{FAULT DETECTION}

The classical way for detecting faults consists of checking the measurable variables of a system in regard to a certain tolerance of the normal values. By detecting the faults in this will help to improve the quality of the parts. The quality assurance is one of the big issues in every manufacturing industry, to meet the competitive marketing world. Distance metric is the main tool for detecting similar images from large databases containing original image. In proposed system, Euclidean distance and histogram comparison is done for the purpose of image matching to detect the fault image. Similarity between the restored image and original image is analyzed based on the Euclidean distance and histogram, then based on the similarity the faulty part is identified. The expression used for the calculation of Euclidean distance [10] is given by:

$\mathrm{ED}=\sqrt{\sum_{\mathrm{i}=1}^{\mathrm{N}}(\mathrm{fq}(\mathrm{i})-\mathrm{fdb}(\mathrm{i}))^{2}}$

Where $\mathrm{fq}(\mathrm{i})$ stands for $\mathrm{i}^{\text {th }}$ extracted image and $\mathrm{fdb}(\mathrm{i})$ for corresponding image in database. Here $\mathrm{N}$ refers to number of images in database. From the equation, Euclidean distance between extracted image and the images present in the database are compared. Thus from the distance of the calculated images in the database, images related to the query image is retrieved. Thus from the key value the similarity of the images are compared and also the fault identified from the images that are simulated. Based on the WNN and amoeba element a new image restoration algorithm is proposed in this paper. Algorithm on image restoration will give a systematic approach to implement the experimental results. Steps involved in the proposed image restoration process as follows:

Step 1: Initializing: From the blurred image, select the part of degraded image which is given as the input and make it as training samples.

Step 2: Preprocessing: Divide the training samples into flat and edge parts by amoeba structural element which is generally used for filtering purposes. (X p, Dp) are vector pairs of flat part and (X 'p, D'p) are vector pairs of edge part divided by the amoeba operator.

Step 3: Restoration: Input the training vector pairs (X p, Dp) and (X 'p, D'p), and train them under parameters adjustment formula of wavelet neural network.

Step 4: Fault Detection: The input signal is first decomposed using some wavelet basis by the neurons in the hidden layer. The wavelet coefficients are then output to one or more summers whose input weights are modified in accordance with some learning algorithm.

Step 5: Network parameters, weight matrix of hidden layer and threshold matrix of output layer can be obtained.

Step 6: Then wavelet neural network model of flat part and edge part can be obtained from the training samples given as the input to the WNN.

Step 7: Divide the degraded image into flat and edge parts by using amoeba operator.

Step 8: Input the flat part and the edge part to neural network model of flat part and edge part respectively which have been trained well. Original image estimation can be obtained from the two training models.

Step 9: Then the simulated image is matched with the original image for identifying defects.

\section{EXPERIMENTAL RESULTS}

The proposed system for the image restoration in industrial applications is implemented in the working platform of MATLAB

The proposed system is executed in three phases: image adjusting, processing and restoration. The input image used for this experiment is shown in figure1.

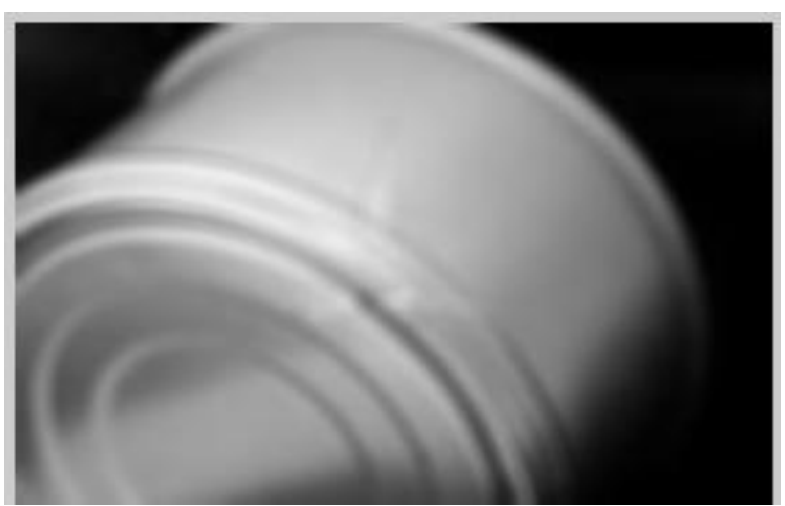

Figure1: Input image sample

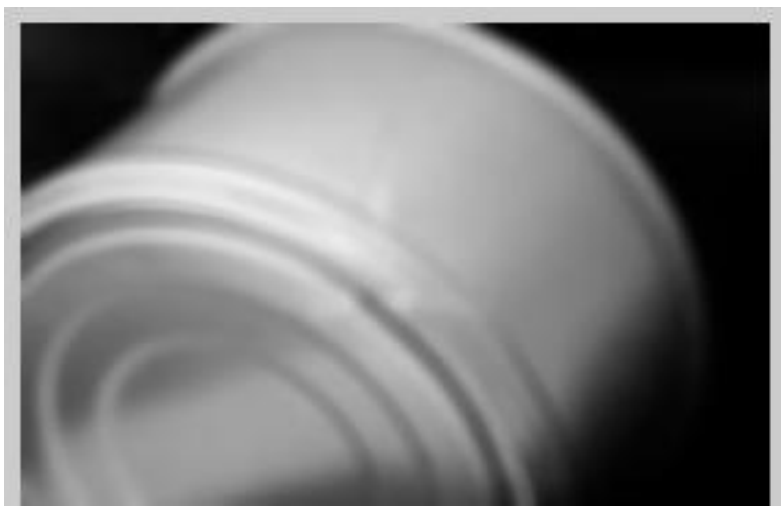

Figure 2: Preprocessed image

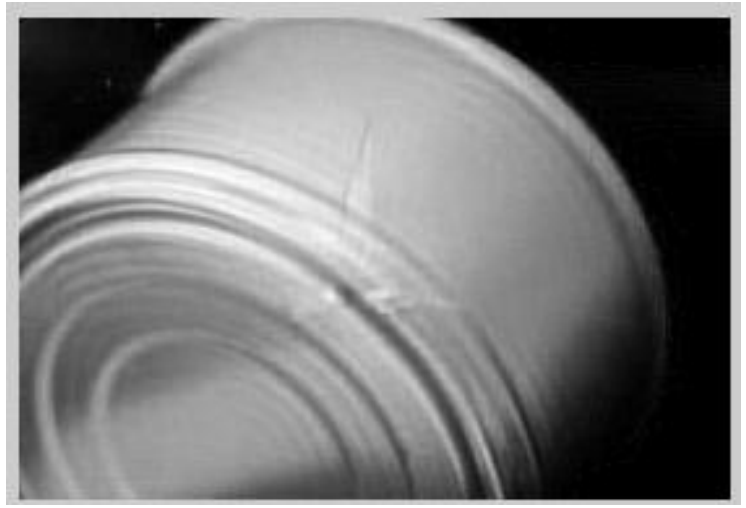

Figure 3: Restored image for identifying defects with original image

The figure 2 shows the preprocessed images, from this image itself it is clear that the blurred regions in the images are removed well and the noise level also is reduced. The further process like segmentation and classification will become easy 
and accurate by using these preprocessed images. The images after the training by WNN are shown in figure 3. The restored image is compared with the original image for identifying any defects.

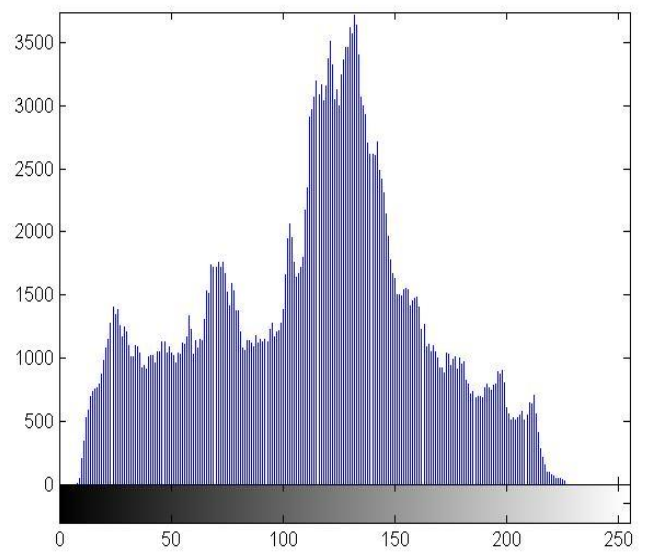

Figure 4: Histogram plot of input image

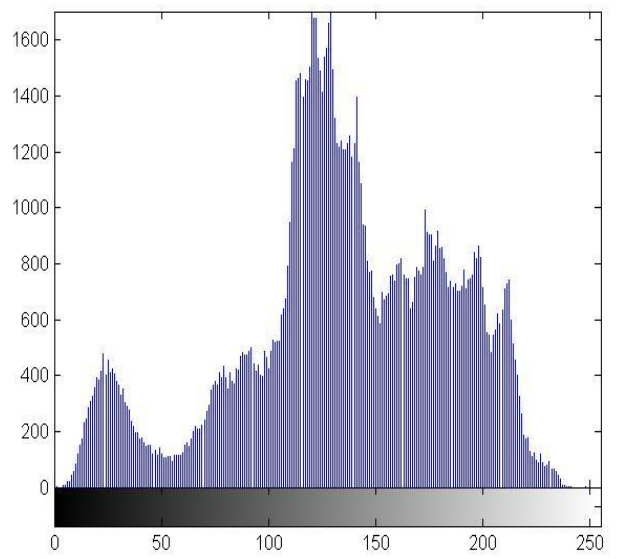

Figure 5: Histogram plot of Output image

By comparing the images one can identify the defects in the simulated image. The restoration result obtained by the proposed WNN is in satisfactory level.

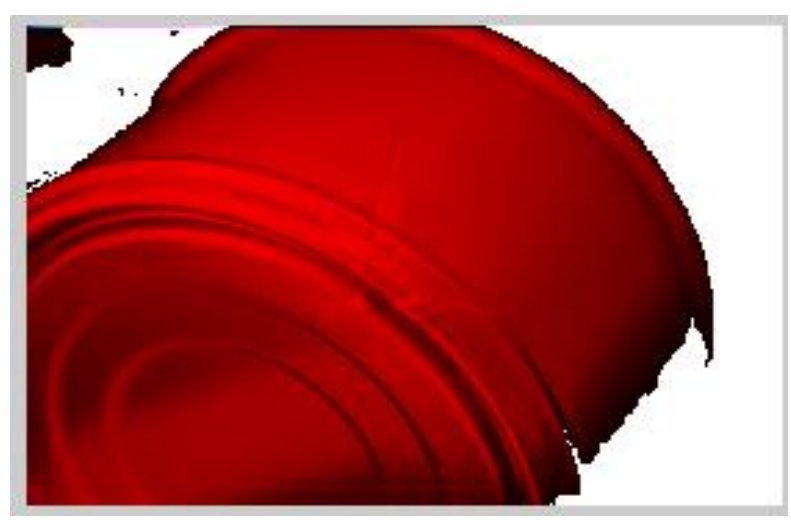

Figure 6: Defective product identification

The speed while using proposed method is $80 \%$ and accuracy while using original image is more than $15 \%$ higher than simulated image. The sensitivity of classifier while using the simulated image is $60 \%$ and in case of original image is $77 \%$.
Hence from this performance analysis it can be shown that the proposed system while using restored image is better than using blurred image. Hence the proposed system becomes well suitable for real time usage.

\section{CONCLUSION}

In order to obtain the quality, reduced cost and weight performance of the automotive system is to be improved. Each and every part should be designed according to its specifications and each system is monitored prudently. WNN proposed in this paper will make huge difference in the field of industries. Image restoration using WNN will detect the defects in the restored image by comparing with the original image with the help of Euclidean distance and histogram comparison. Amoeba element proposed in this paper will automatically adjust shapes and sizes according to the specifications. Hence the image restoration in industries has a great advantage to develop errorless devices.

\section{REFERENCES}

[1] H.Murase and S.K. Nayer, "Visual Learning and recognition of 3-D objects from appearance", International Journal of Computer Vision, Vol. 14, 1994, pp.5-24.

[2] Rasti, B, Sveinsson J.R, Ulfarsson M.O, "Wavelet-Based Sparse Reduced-Rank Regression for Hyper spectral Image Restoration", IEEE Transactions on Geoscience and Remote Sensing, Vol. 52, No. 10, pp. 6688-6698, 2014.

[3] Marnissi Y, Benazza-Benyahia A, Chouzenoux E, Pesquet, J.-C, "Generalized multivariate exponential power prior for wavelet-based multichannel image restoration", in proceedings of 20th IEEE International Conference on Image Processing (ICIP), pp.2402 2406, September 2013.

[4] Ian-Hui Tan, Ao-Chang Pan, Jian Liang, Yong-Hui Huang, Xiao-yan Fan, "A new algorithm for infrared image restoration based on multi-scale morphological wavelet and Hopfield neural network", in proceedings of International Conference on Wavelet Analysis and Pattern Recognition (ICWAPR), pp. 266-270, 2010.

[5] Guerquin-Kern and Matthieu, "A fast wavelet-based reconstruction method for magnetic resonance imaging", IEEE Transactions on Medical Imaging, Vol. 30, No. 9, pp. 1649-1660, 2011.

[6] Premaratne, P and Burnett, I, " Role of wavelet transforms in image restoration", TENCON 2004, IEEE Region 10 Conference, 21-24 November 2004, Vol A, 243-246.

[7] Rojas R., "Neural Networks:A Systematic Introduction", Springer 1996, Berlin.

[8] Rumelhart D. and J. McClelland, "Parallel, Distributed Processing", MIT Press, Cambridge.

[9] Lin and Cheng-Jian, "Wavelet neural networks with a hybrid learning approach", in proceedings of the international journal of Information science and Engineering, Vol. 22, No. 6 pp.1367-1387, 2006.

[10] Kokare, Manesh, B. N. Chatterjee and P. K. Biswas, "Comparison of similarity metrics for texture image retrieval", in proceedings of the international conference on Convergent Technologies for the AsiaPacific Region, Vol. 2, pp. 571-575, 2003. 


\section{AUTHOR PROFILE}

Anoopa Jose Chittilappilly received her B.Tech degree in Electrical \& Electronics Engineering from Kannur University in 2003 and M.E. degree in Applied Electronics from Anna University in 2005. Since 2005 she is working at I.E.S. College of Engineering, Thrissur, Kerala. She is pursuing her Doctoral program at Karpagam University. Her research interests are Image \& Signal Processing.

Dr. Kamalraj Subramaniam, completed his B.E Electronics and Communication Engineering at Karpagam College of
Engineering, Coimbatore in 2007 and Completed M.E VLSI design in Anna University, Chennai in 2009. His research activity mainly focuses on Doctor of Philosophy recommended design and development of an Intelligent Hearing ability level assessment system using Somatosensory stimuli. Currently he is working as Associate Professor and Deputy Head in department of Electronics and Communication Engineering in the domain of Human Machine Interface Cluster at Karpagam Academy of Higher Education, Coimbatore. 